\title{
Spectroscopic Investigations of the Main-Sequence B Stars in the Association Cas OB2 and the Open Cluster Platais 1 (C2128+488)
}

\author{
I.A. Usenko ${ }^{1}$, V.V. Kovtjukh ${ }^{1}$, S.M. Andrievsky ${ }^{1}$, V.G. Klochkova ${ }^{2}$, \\ V.E. Panchuk ${ }^{2}$, S.V. Ermakov ${ }^{2}$ \\ ${ }^{1}$ Department of Astronomy, Odessa State University, Shevchenko Park, \\ 270014, Odessa, Ukraine \\ ${ }^{2}$ Special Astrophysical Observatory of the Russian Academy of Sciences, \\ Nizhnij Arkhyz, 357147, Russia
}

\begin{abstract}
A spectroscopic investigation of four main-sequence stars in the association Cas OB2 (HD 17327A and B, HD 16893, HD 17443) and of two stars in the open cluster Platais 1 (No. 1 (1921) and No. 111 (1600)) was carried out using high-resolution CCD spectra, obtained with echellespectrometer "LYNX" (6-m telescope, Special Astrophysical Observatory, Russian Academy of Sciences). We have determined the atmospheric parameters for the program stars, their projected rotational velocities and elemental abundances in their atmospheres. Two new HgMn stars - HD 17327A and Platais No. 1 - were detected. HD 17443 is not a Be star.
\end{abstract}

\section{Introduction}

The most informative results on galactic chemical evolution can be obtained from the investigation of open clusters and associations. These stellar systems allow us the unique possibility to study evolutionary changes in the chemical composition among genetically connected stars.

In this work we present the results of a spectroscopic investigation of some main-sequence stars in the association Cas OB2 and in the open cluster Platais $1(\mathrm{C} 2128+488)$. The main aim was to determine the atmospheric parameters and chemical composition for the selected stars.

\section{Program stars}

For the observations we selected six stars, four of which are members of the association Cas OB2, and two are members of the open cluster Platais 1 (C2128+488). The members of Cas OB2, HD 17443 (B9 V) and HD 16893 (A3 Vp), are situated within the strip of main-sequence stars. The secondary component of the visual binary $\mathrm{ADS} 2142-\mathrm{HD} 17327 \mathrm{~B}(\mathrm{~A} 2 \mathrm{Vn}$ ) is also a main-sequence star, while the primary HD 17327A (B8 III) has its position near to the "turn-off" point of this association. HD 17443 is a possible candidate for a Be type star. 
From the open cluster Platais (C2128+488) (Turner et al. 1994) we have selected two B-stars, No. 111 (Platais 1600, B9.5 V) which is a main sequence object, while No. 1 (Platais 1921, B9 IIIp) is situated in the vicinity of the "turn-off" point.

\section{Observations and method of analysis}

The high-resolution spectra of these stars have been obtained with echellespectrometer PFES, equipped with a $1040 \times 1170$ pixel CCD (Panchuk et al. 1998), installed on the 6-m telescope of Special Astrophysical Observatory, Russian Academy of Sciences (Russia, Northern Caucasus). The resolving power was 24000 , with $\mathrm{S} / \mathrm{N} \approx 70-100$. Information concerning the program star spectra is given in Table 1.

Table 1. Program stars spectra

\begin{tabular}{|c|c|c|c|c|c|c|c|c|}
\hline Star & $\bar{V}$ & $\begin{array}{c}\text { HJDD } \\
2450000+\end{array}$ & $\begin{array}{c}\text { Region } \\
(\AA)\end{array}$ & $\begin{array}{c}\text { Exposure } \\
\text { (min) }\end{array}$ & $\begin{array}{c}v \sin i \\
\left(\mathrm{~km} \mathrm{~s}^{-1}\right)\end{array}$ & $\bar{T}_{\text {eff }}$ & $\log g$ & $\bar{V}_{t}$ \\
\hline $\begin{array}{l}\text { HD } 16893 \\
\text { HD } 17327 a\end{array}$ & $\begin{array}{l}8.53 \\
7.53\end{array}$ & $\begin{array}{l}1006.528 \\
1003.527 \\
\mathbf{1 2 4 6 . 1 7 2} \\
\mathbf{1 2 4 6 . 1 7 9}\end{array}$ & $\begin{array}{l}4682-8596 \\
4420-7767 \\
\mathbf{4 3 8 3}-7960 \\
\mathbf{4 3 8 3}-7960\end{array}$ & $\begin{array}{c}20 \\
20 \\
\mathbf{3} \\
\mathbf{7}\end{array}$ & $\begin{array}{l}25 \\
40\end{array}$ & $\begin{array}{l}8500 \\
11700\end{array}$ & $\begin{array}{l}4.00 \\
3.20\end{array}$ & $\begin{array}{l}3.0 \\
1.0\end{array}$ \\
\hline $\begin{array}{l}\text { HD 17327b } \\
\text { HD } 17443\end{array}$ & $\begin{array}{r}10.33 \\
8.74\end{array}$ & $\begin{array}{l}1246.202 \\
1003.480\end{array}$ & $\begin{array}{l}\mathbf{4 3 8 3}-7960 \\
\mathbf{4 4 2 0}-\mathbf{7 7 6 7}\end{array}$ & $\begin{array}{l}30 \\
30\end{array}$ & $\begin{array}{l}200 \\
180\end{array}$ & $\begin{array}{l}10000 \\
10900\end{array}$ & $\begin{array}{l}4.00 \\
4.05\end{array}$ & $\begin{array}{l}3.0 \\
3.0\end{array}$ \\
\hline $\begin{array}{l}\text { No. } 1(1921) \\
\text { No. } 111(1600)\end{array}$ & $\begin{array}{l}11.53 \\
12.54\end{array}$ & $\begin{array}{l}1003.304 \\
1004.461\end{array}$ & $\begin{array}{l}4420-7767 \\
4420-7767\end{array}$ & $\begin{array}{l}30 \\
54\end{array}$ & $\begin{array}{c}15 \\
150\end{array}$ & $\begin{array}{l}10000 \\
13000\end{array}$ & $\begin{array}{l}3.50 \\
4.00\end{array}$ & $\begin{array}{l}3.5 \\
3.0\end{array}$ \\
\hline
\end{tabular}

The CCD spectra have been reduced using the DECH20 code (Galazutdinov 1992). The analysis has been carried out using Kurucz' (1992) WIDTH9 code. Atmosphere models have been interpolated from Kurucz's (1992) grid. Oscillator strengths were taken from Kurucz (1995) database (CD-ROMs 15, 18). For the star HD 16893 we also used additional data from Adelman et al. (1996).

To evaluate line blends in the spectra of stars with high projected rotational velocities, the spectrum synthesis technique was applied. This was performed with the help of the SYNSPEC code (Hubeny et al. 1994). The projected rotational velocities for the program stars were estimated by fitting the synthesized spectrum to the observed one. Results are given in Table 1.

\section{Atmospheric parameters and chemical composition}

We used several approaches to estimate the atmosphere parameters of the program stars. Among them: $(U-B),(B-V)-\mathrm{T}_{\text {eff }}, \log g$ and $(b-y)-T_{\text {eff }}, \log g$ calibrations from Castelli (1991), $(b-y), m 1, c 1, \beta-T_{\text {eff }}, \log g$ calibrations from Moon \& Dworetsky (1985); and comparisons of the observed $\mathrm{H} \alpha$ and $\mathrm{H} \beta$ line profiles with the synthetically generated ones.

For the coolest star of our sample, HD 16893, the surface gravity was determined by forcing $\mathrm{Fe}$ I and Fe II to produce the same abundance. Its microturbulent velocity was obtained by forcing the abundances from Fe I lines to be independent of the equivalent line widths. In case of HD17327A and Platais No. 1 (1921) for the determination of $V_{t}$ we have used Fe II lines. For the rest 
Table 2. Abundances for HD 16893.

\begin{tabular}{lrrrrrr}
\hline Element & \multicolumn{3}{c}{$\log g f$ (Kurucz) } & \multicolumn{3}{c}{$\log g f$ (4delman et al.) } \\
\cline { 2 - 7 } & {$[\mathrm{EI} / \mathrm{H}]$} & \multicolumn{1}{c}{$\sigma$} & $\mathrm{NL}$ & \multicolumn{1}{c}{ EI/H] } & \multicolumn{1}{c}{$\sigma$} & \multicolumn{1}{c}{ NL } \\
\hline C I & -0.45 & 0.17 & 7 & -0.50 & 0.18 & 5 \\
N I & +0.09 & 0.03 & 2 & -0.20 & 0.51 & 5 \\
O I & -0.12 & 0.00 & 1 & -0.07 & 0.18 & 4 \\
Na I & +0.29 & 0.00 & 1 & - & - & - \\
Mg I & -0.98 & 0.22 & 2 & -0.58 & 0.00 & 1 \\
Mg II & - & - & - & +0.54 & 0.00 & 1 \\
Al I & +0.92 & 0.08 & 2 & +0.92 & 0.08 & 2 \\
Si II & -0.45 & 0.00 & 1 & -0.14 & 0.38 & 3 \\
S I & +0.20 & 0.20 & 3 & +0.20 & 0.20 & 3 \\
Ca I & -0.02 & 0.23 & 15 & - & - & - \\
Sc II & +0.04 & 0.38 & 6 & -0.12 & 0.12 & 2 \\
Ti I & - & - & - & +0.37 & 0.26 & 2 \\
Ti II & -0.20 & 0.15 & 13 & -0.05 & 0.28 & 13 \\
V II & +0.78 & 0.00 & 1 & +0.78 & 0.00 & 1 \\
Cr II & -0.09 & 0.15 & 10 & -0.03 & 0.24 & 10 \\
Mn I & +0.65 & 0.24 & 4 & - & - & - \\
Mn II & - & - & - & +1.05 & 0.02 & 2 \\
Fe I & -0.21 & 0.17 & 18 & -0.68 & 0.00 & 1 \\
Fe II & -0.17 & 0.09 & 10 & -0.14 & 0.42 & 13 \\
Co I & +1.10 & 0.18 & 5 & +1.19 & 0.26 & 6 \\
Ni I & +0.06 & 0.20 & 8 & - & - & - \\
Cu I & +0.22 & 0.00 & 1 & +0.22 & 0.00 & 1 \\
Zn I & -0.01 & 0.27 & 2 & - & - & \\
Sr I & +1.43 & 0.00 & 1 & - & - & \\
Y I & +2.56 & 0.00 & 1 & - & - & \\
Y II & +0.58 & 0.49 & 5 & +0.41 & 0.34 & 3 \\
Zr II & +0.63 & 0.28 & 3 & +0.63 & 0.28 & 3 \\
Ba II & +0.23 & 0.42 & 4 & +0.35 & 0.00 & 1 \\
La II & +1.14 & 0.01 & 2 & +1.14 & 0.01 & 2 \\
Ce II & +1.21 & 0.43 & 3 & +1.43 & 0.56 & 4 \\
Pr II & - & - & - & +1.72 & 0.00 & 1 \\
Nd II & +1.85 & 0.00 & 1 & +1.85 & 0.00 & 1 \\
Sm II & +1.90 & 0.00 & 1 & - & - & - \\
\hline NL - number of lines & & & & & \\
& & & & & &
\end{tabular}

of the program stars a microturbulent velocity of $3 \mathrm{~km} \mathrm{~s}^{-1}$ was adopted as a more appropriate value for the late B stars. All the finally adopted atmospheric parameters are listed in Table 1 . In Tables 2 and 3 we give the calculated abundances for HD 16893, HD 17327A and Platais No. 1 (1921), respectively.

It should be noted that for the rapidly rotating $B$ stars HD 17443 and Platais No. 111 (1600) we estimated only the helium and magnesium abundance from two strong features He I $4471 \AA$ and Mg II $4481 \AA$. This was performed using spectral synthesis. In both cases the helium and magnesium abundances appeared to be solar.

Our abundance analysis revealed the following.

1. two stars, HD 17327A and Platais No. 1, appeared to be mercurymanganese stars. Their chemical composition is typical for that kind of stars: a strong overabundance of $\mathrm{Mn}$ and $\mathrm{Hg}$ accompanied by the overabundances of $\mathrm{C}$, $\mathrm{S}, \mathrm{P}, \mathrm{Y}$ and $\mathrm{Zr}$. This overabundance could be explained by the light-inductive drift mechanism (LID) (Atutov \& Shalagin, 1988)

2. HD 16893 also demonstrates some Mn overabundance, but its mercury content was not measured. The final classification of this star requires therefore additional investigation.

3. an interesting result was obtained for the helium content. This element is overabundant in the atmospheres of HD 17327A and Platais No. 1 (1921), while 
Table 3. Abundances for HD 17327A and Platais No. 1 (1921).

\begin{tabular}{|c|c|c|c|c|c|c|c|c|c|c|c|c|}
\hline \multirow{3}{*}{ Element } & \multicolumn{9}{|c|}{ HD 17327a } & \multirow{2}{*}{\multicolumn{3}{|c|}{ Platais No. 1 (1921) }} \\
\hline & \multicolumn{3}{|c|}{1} & \multicolumn{3}{|c|}{2} & \multicolumn{3}{|c|}{3} & & & \\
\hline & {$[\mathrm{El} / \mathrm{H}]$} & $\sigma$ & NL & {$[\mathrm{E} 1 / \mathrm{H}]$} & $\bar{\sigma}$ & $\mathrm{NL}$ & $[\mathrm{E}] / \mathrm{H}]$ & $\sigma$ & $\mathrm{NL}$ & El/H] & $\sigma$ & NL \\
\hline $\mathrm{He} \mathrm{I}$ & +0.17 & 0.57 & 2 & +0.40 & 0.00 & 1 & +0.20 & 0.00 & 1 & +0.79 & 0.12 & 2 \\
\hline $\mathrm{CI}$ & +0.78 & 0.00 & 2 & +0.41 & 0.00 & 1 & +0.47 & 0.31 & 3 & +0.42 & 0.38 & 2 \\
\hline & & & & +0.31 & 0.00 & 1 & & & & -0.48 & 0.00 & 1 \\
\hline O I & -0.39 & 0.32 & 2 & -0.10 & 0.32 & 5 & +0.02 & 0.44 & 3 & +0.37 & 0.28 & 4 \\
\hline $\mathrm{Mg} \mathrm{I}$ & -0.56 & 0.00 & 1 & -0.46 & 0.00 & 1 & & & & -0.35 & 0.21 & 3 \\
\hline Mg II & +0.08 & 0.00 & 1 & & & & -0.31 & 0.44 & 3 & +0.24 & 0.00 & 1 \\
\hline Al II & -0.36 & 0.00 & 1 & & & & & & & & & \\
\hline Si II & -0.63 & 0.33 & 6 & -0.75 & 0.20 & 6 & -0.43 & 0.24 & 6 & -0.73 & 0.34 & 5 \\
\hline P II & +0.47 & 0.00 & 1 & +1.11 & 0.00 & 1 & +1.48 & 0.00 & 1 & +1.54 & 0.69 & 2 \\
\hline S II & +0.20 & 0.05 & 3 & +0.46 & 0.32 & 3 & +0.43 & 0.00 & 1 & & & \\
\hline Sc II & +1.25 & 0.00 & 1 & +1.35 & 0.00 & 1 & & & & +0.64 & 0.35 & 2 \\
\hline Ti II & +0.71 & 0.28 & 9 & +0.45 & 0.40 & 4 & +0.40 & 0.51 & 4 & -0.08 & 0.28 & 10 \\
\hline $\begin{array}{l}\text { Cr II } \\
\text { Mn I }\end{array}$ & +0.47 & 0.26 & 15 & +0.46 & 0.31 & 13 & +0.44 & 0.36 & 9 & +1.08 & 0.18 & 10 \\
\hline Mn II & +1.94 & 0.20 & 18 & +2.01 & 0.24 & 11 & +1.73 & 0.24 & 14 & $\begin{array}{l}+1.65 \\
+1.66\end{array}$ & $\begin{array}{l}0.00 \\
0.18\end{array}$ & 12 \\
\hline $\mathrm{Fe}$ II & -0.09 & 0.26 & 66 & -0.08 & 0.25 & 32 & -0.14 & 0.21 & $\begin{array}{l}14 \\
26\end{array}$ & $\begin{array}{r}+0.13 \\
\end{array}$ & 0.26 & 23 \\
\hline Y II & +2.51 & 0.00 & 1 & +1.57 & 0.38 & 2 & +1.84 & 0.00 & 1 & +1.16 & 0.35 & 3 \\
\hline $\mathrm{Zr}$ II & +4.57 & 000 & 1 & +420 & 0.00 & 1 & & & & +2.02 & 0.50 & 2 \\
\hline Hg 1 & & 0.00 & & & & & & & & +4.05 & 0.00 & \\
\hline
\end{tabular}

its content in Platais No. 111 and HD 17443 is normal. It should be noted that HD 17327A and Platais No. 1 (1921) have low projected rotational velocities that might be favorable to the LID mechanism in atmospheres. As a result, we have obtained the helium overabundance.

4. HD 17443 is not likely to be a Be star because we did not detect any emission features in $\mathrm{H} \alpha$ line profile.

\section{References}

Adelman, S.J., Philip, A.G.D., Adelman, C.J. 1996, MNRAS 282, 953

Atutov, S.N., Shalagin, A.M., 1988, Soviet Ast.Lett. 14, 664

Castelli, F., 1991, A\&A 251, 106

Galazutdinov, G.A. 1992, Prepr. SAO RAS No. 92

Hubeny, I., Lanz, T., Jeffrey, C.S. 1994, Newsletter Analys. Astron. Spec. No. 20,30

Kurucz, R.L. 1995, Laboratory and Astronomical High Resolution Spectra, Eds.

A.J. Sauval, R. Blomme, N. Grevesse, ASP Conf. Ser. 81, 595

Kurucz, R.L. 1992, The Stellar Populations of Galaxies Eds. B. Barbuy, A. Renzini, IAU Symp. 149, 225

Moon, T.T., Dworetsky, M.M., 1985, MNRAS 217, 305

Panchuk, V.E., Najdenov, I.D., Klochkova, V.G., Ivanchik, A.B., Yermakov, S.V., Murzin, V.A. 1998, Bull. SAO RAS 44, 127

Turner, D.G., Evans, N.R. 1984, ApJ 283, 254

Turner, D.G., Mandushev, G.I., Forbes, D. 1994, AJ 107, 1796 